\title{
Does early initiation of therapeutic plasma exchange improve outcome in pediatric stem cell transplant-associated thrombotic microangiopathy?
}

\author{
Sonata Jodele, Benjamin L. Laskin, Jens Goebel, Jane C. Khoury, Susan L. Pinkard, \\ Patricia M. Carey, and Stella M. Davies \\ Division of Bone Marrow Transplantation and Immune Deficiency, the Division of Nephrology and \\ Hypertension, and the Division of Biostatistics and Epidemiology, Cincinnati Children's Hospital \\ Medical Center; and Hoxworth Blood Center, University of Cincinnati, Cincinnati, Ohio
}

\begin{abstract}
BACKGROUND-The use of therapeutic plasma exchange (TPE) in hematopoietic stem cell transplant-associated thrombotic microangiopathy (TA-TMA) is controversial because the exact mechanism of injury in TA-TMA is not yet understood.
\end{abstract}

STUDY DESIGN AND METHODS—The study objective was to retrospectively review the outcome of children receiving TPE for TA-TMA at our institution. We hypothesized that patients initiating TPE earlier in their disease course would receive a greater benefit than those starting later, regardless of the therapeutic mechanism.

RESULTS-We identified 10 consecutive pediatric patients with TA-TMA treated with TPE. Nine of these patients showed normalization of the laboratory variables associated with microangiopathy during their TPE course, but only five patients recovered renal function and survived TA-TMA. The five survivors started TPE a median of 17 days (range, 4-25 days) after TA-TMA diagnosis while the five patients who died started TPE a median of 32 days (range, 1773 days) after TA-TMA was diagnosed. Three of the five survivors had multiorgan failure at TATMA diagnosis and completely recovered with early institution of TPE. These three survivors were able to discontinue renal replacement therapy, and all achieved a normal posttreatment creatinine. The five patients with later institution of TPE progressed to end-stage renal disease and all died. There were no serious TPE-related complications in either group.

CONCLUSION-This is the first report evaluating TPE response in regard to procedure initiation time after TA-TMA diagnosis. Our data suggests that early initiation of TPE might be beneficial even in patients with multiorgan failure due to TA-TMA.

Address reprint requests to: Sonata Jodele, MD, Division of Bone Marrow Transplant and Immune Deficiency, Cincinnati Children's Hospital Medical Center, 3333 Burnet Avenue, MLC 7015, Cincinnati, OH 45229; sonata.jodele@cchmc.org.

The Cincinnati Children's Hospital and Medical Center Institutional Review Board exempted this study from obtaining informed consent from the patient and family.

CONFLICT OF INTEREST

There are no conflicts of interest to disclose from any of the authors. 
Hematopoietic stem cell transplant (HSCT)-associated thrombotic microangiopathy (TATMA) presents as de novo microangiopathic hemolytic anemia, thrombocytopenia, and renal dysfunction. TA-TMA is a significant complication of HSCT with a reported acute mortality of more than $60 \%$ and a high prevalence of chronic kidney disease in survivors. ${ }^{1,2}$ The diagnosis of TA-TMA is challenging and requires a high index of suspicion. ${ }^{3}$ Our limited understanding of the exact mechanism of injury and pathogenesis in HSCT patients hampers the development of optimal therapies. Chemoradiotherapy, calcineurin inhibitors, viral infections, and graft-versus-host disease (GVHD) have each been implicated as risk factors for TA-TMA with the resulting endothelial damage representing the final common pathway of injury. ${ }^{4}$

Therapeutic plasma exchange (TPE) has been reported as successful first-line therapy in other thrombotic microangiopathies including atypical hemolytic uremic syndrome (aHUS), adult-onset diarrhea-positive HUS, and thrombotic thrombocytopenic purpura (TTP). ${ }^{5-7}$ However, the use of TPE in TA-TMA remains controversial. Previous studies have concluded that TPE is less effective in the treatment of TA-TMA compared to TTP. ${ }^{8}$ The first large randomized trial for TTP comparing empiric therapy with TPE versus plasma infusions was carried out by Canadian Apheresis Study Group and showed clear superiority of TPE therapy in this disease. ${ }^{9}$ Patients with TTP likely benefit from TPE because the therapy restores von Willebrand factor-cleaving protease activity (ADAMTS13), either through replacement of the deficient protein and/or through removal of inhibitory antibodies. Decreased ADAMTS13 activity has not been shown to be involved in the pathogenesis of TA-TMA. ${ }^{10}$ The majority of patients with aHUS have documented complement system abnormalities, including complement factor $\mathrm{H}$ autoantibody and CFHR1 gene defects that respond well to therapy with rituximab and TPE. ${ }^{11,12}$ Due to clinical and histologic similarity of TA-TMA and aHUS, rituximab therapy has been tried in HSCT patients with successful reversal of microangiopathy reported in small cohorts of patients, although without clear understanding of mechanism of action in transplant population. ${ }^{13-15}$

Our treatment approach for TA-TMA at the time was to first withdraw or reduce calcineurin inhibitor therapy, if possible, and substitute it with mycophenolate mofetil (MMF). Next, a therapeutic trial of rituximab was given to patients with continued signs of microangiopathy or in those whom potentially offending agents could not be eliminated. First, the rituximab dose was given at TA-TMA diagnosis, followed by two to four weekly doses of $375 \mathrm{mg} / \mathrm{m}^{2}$ / dose. Rituximab is considered an early noninvasive therapeutic option that is readily available with the potential to abort TA-TMA in early stages. TPE is initiated in patients not responding to the above measures or developing signs of severe multiorgan involvement at the discretion of their primary physician. Early reports in the literature describing the lack of benefit of TPE in TA-TMA, along with its suggested association with increased mortality and procedure-related complications often discourage physicians from considering TPE as a therapeutic option for TA-TMA. ${ }^{16}$ Several recent reports have shown the benefit of early TPE initiation in TA-TMA. ${ }^{15,17-19}$ In our retrospective review we hypothesized that patients starting TPE early in the course of their disease, before the development of irreversible organ damage, would have a better outcome compared to those starting later regardless of the therapeutic mechanism. 


\section{MATERIALS AND METHODS}

We reviewed the records of all consecutive HSCT patients listed in the transplant database as treated with TPE for diagnosis of TA-TMA at our institution between January 2009 and June 2010. In all these patients TA-TMA diagnosis was made by the treating HSCT physician during posttransplant follow-up time using uniform published diagnostic criteria: de novo microangiopathic anemia with the presence of schistocytes on peripheral blood smear, de novo thrombocytopenia, elevated lactate dehydrogenase (LDH), low haptoglobin, doubling of serum creatinine, and/or evidence of neurologic symptoms. ${ }^{8}$ Due to age-dependent laboratory norms for hemoglobin $(\mathrm{Hb}), \mathrm{LDH}$, and serum creatinine, these tests were reported as abnormal for each patient based on their values. Elevation in serum creatinine was reported as doubling of serum creatinine from pretransplant value for each patient. Neurologic symptoms were identified as documented change in mental status or seizures coinciding with diagnosis of TA-TMA.

Identified patients were divided into two groups: survivors and nonsurvivors, defined by their status at 21 months after HSCT. The 21-month time point was chosen based on expiration of the last patient in the non-survivor group and follow-up of patients in the survivor group past this time point. Demographic information, transplant characteristics, therapy, complications, and outcomes were compared in these two groups.

TPE was performed using a single-volume plasma exchange with an apheresis system (Spectra Optia, Caridi-anBCT, Lakewood, CO). Daily TPE was continued until resolution of microangiopathy (see definition below) and tapered thereafter using the following schedule.

First, TPE was decreased to every other day for approximately 2 weeks, decreased to twice a week, and then finally discontinued. If laboratory findings indicated recurrence of microangiopathy the tapering was stopped and TPE resumed at the previous frequency.

Due to the volume of the extracorporeal circuit, priming was done with red blood cells (RBCs) for patients weighing less than $21 \mathrm{~kg}$. Replacement fluid was calculated based on the patient's total blood volume. Fresh-frozen plasma (FFP) was replaced at $100 \%$ leaving the patient euvolemic at the end of the procedure. Anticoagulation was achieved using citrate. Prophylactic infusion of calcium gluconate was administered for the patient to avoid citrate toxicity.

For patients receiving weekly rituximab during TPE therapy, rituximab was administered immediately after the TPE session. ${ }^{20}$ TA-TMA monitoring included daily complete blood counts and examination of peripheral blood smears for the presence of schistocytes, serum LDH and creatinine, and weekly haptoglobin. Screening for disseminated intravascular coagulation or the presence of RBC or platelet (PLT) autoantibodies was performed at the time of TA-TMA diagnosis and/or later as clinically indicated. Patients with evidence of proteinuria on weekly screening urinalyses had random urine protein-to-urine creatinine ratios checked. Ratios of more than $0.2 \mathrm{mg} / \mathrm{mg}$ indicated subnephrotic range proteinuria, and ratios of more than $2 \mathrm{mg} / \mathrm{mg}$ identified subjects with nephrotic range proteinuria.

Resolution of microangiopathy was defined as normalization of LDH and haptoglobin and disappearance of schistocytes. Complete resolution of TA-TMA was defined as resolution of 
microangiopathy and acute kidney injury (return of the serum creatinine to pretransplant baseline without renal replacement therapy). Hypertension was defined as a systolic or diastolic blood pressure over the 95th percentile for age, sex, and height or the need for antihypertensive therapy. ${ }^{21}$ We reported stabilization of blood counts as favorable outcomes, but due to different transfusion variables and the presence of PLT antibodies in some patients, $\mathrm{Hb}$ and PLT count were not included in the response criteria for our analysis.

Routine post-HSCT monitoring included surveillance for cytomegalovirus (CMV), EpsteinBarr virus, and adenovirus by weekly or twice weekly blood polymerase chain reaction testing in blood. Standard prophylaxis included monthly pentamidine for Pneumocystis jirovecii and anti-fungal prophylaxis with weekly ambisome, daily voriconazole, or daily caspofungin. Acyclovir prophylaxis was given if patient or donor was seropositive for CMV or herpes simplex virus. All allogeneic transplant recipients initially received cyclosporine for GVHD prophylaxis with target blood trough levels of 250 to $350 \mathrm{ng} / \mathrm{mL}$. The study was approved by the institutional review board and exempted from the requirement of obtaining informed consent due to its retrospective nature.

\section{Statistical analysis}

We compared those surviving after a diagnosis of TA-TMA with those who died. Clinical and treatment characteristics of each group were compared using Fisher's exact test for categoric variables and Wilcoxon rank sum for most continuous variables. Life table analysis was used, with the Wilcoxon test, for two variables: number of days to resolution of microangiopathy and number of days for creatinine to return to pre-HSCT baseline. This was due to nonresolution at the time of final follow-up of death. A p value of less than 0.05 was considered significant. Analyses were performed using computer software (SAS, Version 9.2, SAS Institute, Cary, NC).

\section{RESULTS}

\section{Patient characteristics}

A total of 155 HSCTs (113 allogeneic and 42 autologous) were performed at our institution between January 1, 2009, and June 30, 2010. Ten consecutive patients were treated with TPE for TA-TMA: five survived TA-TMA and the other five died. There were no differences in patient demographics or transplant characteristics between the two groups (Table 1). Seven of the 10 identified patients received an allogeneic transplant and the remaining three transplants were autologous. TA-TMA was diagnosed within 100 days after HSCT in 9 of 10 patients. The 10th patient had a later presentation of TA-TMA ( $>200$ days after HSCT) coinciding with CMV viremia. Concomitant viral infections that preceded TA-TMA were similar in both groups. There were no bacterial or fungal infections documented before starting TPE (Table 1). All patients had hypertension requiring medical management. Before the diagnosis of TA-TMA, patients in both groups had similar renal function as measured by creatinine and a formal nuclear glomerular filtration rate (nucGFR, 99Tc-DTPA; Table 1). Three of the 10 patients had a renal biopsy, confirming the diagnosis of TA-TMA. TA-TMA features are listed in Table 2. 


\section{TA-TMA-directed therapy}

After the diagnosis of TA-TMA, calcineurin inhibitor cyclosporine was withdrawn in six of the seven patients receiving an allogeneic transplant and substituted with MMF, and one patient received reduced cyclosporine dose. Eight of the 10 patients received two to four weekly rituximab doses without resolution of microangiopathic anemia and proceeded to TPE at the discretion of the primary physician. The remaining two patients, both in the survivor group, presented with acute TA-TMA and received concomitant rituximab and TPE. Two patients in the survivor group and three patients in the nonsurvivor group received additional rituximab therapy for PLT antibodies after initiation of TPE. De novo PLT antibodies were detected in these patients between 42 and 100 days after TA-TMA diagnosis.

\section{Overall TA-TMA outcome}

In the entire cohort, single volume daily TPE was initiated 4 to 73 days after TA-TMA diagnosis, continued until resolution of microangiopathy and then tapered as described above. Even though 9 of $10(90 \%)$ patients showed laboratory resolution of microangiopathy during TPE, only five patients (50\%) recovered renal function and survived at a median follow-up of 997 days after HSCT (range, 689-1249 days). The five survivors started TPE a median of 17 days (range, 4-25 days) after a diagnosis of TA-TMA, while the five patients who died started TPE a median of 32 days (range, 17-73 days) after diagnosis $(\mathrm{p}=0.11$ ).

All of the five survivors required fewer sessions of TPE (median, 25 vs. $69 ; \mathrm{p}=0.01$ ) to control microangiopathic hemolysis. In surviving patients, laboratory signs of microangiopathy resolved within 16 days and creatinine had normalized at a median of 40 days after starting TPE. In contrast, and despite prolonged courses of TPE, one patient in the nonsurvivor group died with active signs of microangiopathy, while the other four experienced resolution of laboratory signs of microangiopathy at a median of 50 days after starting TPE therapy. However, these four patients continued to require renal replacement therapy and died at a median of 256 days after HSCT (range, 151-631 days; Table 2). Four of five patients died due to end-stage renal disease (ESRD). One patient after autologous stem cell transplantation died of secondary myelodysplastic syndrome while on chronic dialysis for ESRD.

\section{Survivor group}

Three of five patients in the survivor group had multiorgan failure at the time when TPE was started for the diagnosis of TA-TMA. Two patients with acute TA-TMA after allogeneic transplant were started on TPE and rituximab 4 days after TA-TMA diagnosis and fully recovered organ function after 14 and 16 days of TPE. The third patient, an autologous transplant recipient was given two doses of rituximab, had histologic evidence of TA-TMA by renal biopsy, and was started on TPE 17 days after TA-TMA presentation. All three survivors with multiorgan failure required renal replacement therapy at the time of TA-TMA diagnosis and received hemodialysis for 7, 19, and 30 days, respectively. The remaining two patients in the survivor group had severe hypertension, posterior reversible encephalopathy syndrome with seizures, and renal insufficiency, but did not require dialysis. These two patients started TPE after failing to have a complete response to four doses of rituximab. 
Four survivors had posttransplant assessment of renal function by nucGFR after completion of TPE that demonstrated a median glomerular filtration rate of $95 \mathrm{~mL} / \mathrm{min} / 1.73 \mathrm{~m}^{2}$ (Table 2).

\section{Nonsurvivor group}

Two of five patients in the nonsurvivor group had multi-organ failure at the time when TPE was started for a diagnosis of TA-TMA. Three patients developed severe polyserositis; one required emergent pericardiocentesis for impending tamponade. Three of these five patients were receiving hemodialysis when TA-TMA was diagnosed and the fourth patient was started on renal replacement therapy later. The fifth patient also developed renal failure but the family declined hemodialysis before his death. All five patients in the nonsurvivor group received weekly rituximab before TPE without adequate control of TA-TMA. Three of them continued rituximab therapy after initiation of TPE for documented PLT antibodies. None of these five patients recovered renal function before their death.

None of the patients receiving an allogeneic transplantation developed acute GVHD. The two patients after allogeneic HSCT with severe polyserositis were presumed to have chronic GVHD, but neither patient had evidence of skin, gastrointestinal, or liver GHVD. These patients remained on MMF therapy. One patient with polyserositis had received an autologous HSCT.

\section{Complications of TPE}

One infection with coagulase negative Staphylococcus occurred in the catheter used for TPE and hemodialysis in a terminally ill patient several days before his death. There were no other TPE procedure- or central line-related complications.

\section{DISCUSSION}

The benefit of TPE in the treatment of TA-TMA remains unknown due to variable outcome measurements, an incomplete understanding of possible therapeutic mechanisms, and a lack of controlled trials. Reports on the use of TPE in TA-TMA have only included case series and retrospective reviews. Blood and bone marrow transplant (BMT) consensus guidelines on TA-TMA have reported poor responses and high mortality rates in patients treated with TPE from 1991 to 2003. The authors summarized 11 studies each including more than five patients and reported a median response rate of $36.5 \%$ (range, $0 \%-80 \%$ ) with an associated mortality of $80 \%$ (range, $44 \%-100 \%$ ). ${ }^{8}$ The authors acknowledged that the high mortality rates may have been biased by including only the sickest patients. ${ }^{1,10}$ In our recent comprehensive review on TA-TMA we summarized the most current literature (2003current) on the use of TPE with a median response rate of 59\% (range, 27\%-80\%), albeit in uncontrolled, heterogeneous populations. ${ }^{4}$

Cho and colleagues ${ }^{19}$ validated the current diagnostic criteria for TA-TMA and reported that therapy with TPE, defibrotide, and/or discontinuation or reduction of calcineurin inhibitors had the greatest benefit in patients with "probable TMA" with reported response rates to therapy as high as $74 \%$. These patients with probable TMA did not have evidence of renal injury (defined as a doubling of serum creatinine) or neurologic deficits at the time of TA- 
TMA diagnosis, suggesting that therapy should be considered early, as soon as TA-TMA is suspected, to avoid irreversible organ damage. Other reports, notably in patients with fulminant TA-TMA, have also suggested that prompt initiation of TPE may be associated with favorable outcomes. ${ }^{15}$

To date, only one prospective trial has assessed the therapeutic benefit of TPE in TA-TMA. Worel and coworkers ${ }^{17}$ reported responses of $64 \%$ (7/11) in patients diagnosed with TATMA who were treated with immediate withdrawal of cyclosporine and initiation of TPE. TPE was performed daily and was tapered after normalization of LDH levels, an increase in PLT count, and clinical improvement. Responders showed improvement between 11 and 35 days after initiation of TPE and received on average 13 (range, 10-27) TPE sessions. The authors concluded that early initiation of therapy may provide benefit.

To the best of our knowledge, no prior study has evaluated the response to TPE based on the time to initiate therapy after the diagnosis of TA-TMA. At our institution TPE was offered only to patients not responding to other clinical measures like reduction of calcineurin inhibitor exposure or use of rituximab except for two patients with acute multiorgan failure where all therapeutic measures were implemented at once. While TA-TMA was diagnosed at a similar time after transplantation in all but one patient with late presentation of disease, the time to initiate TPE was influenced by primary BMT physician decision since there was no universally accepted agreement for use of TPE in TA-TMA. Even though microangiopathy resolved in $90 \%$ of the patients receiving TPE in this cohort, only 50\% of patients starting TPE earlier fully recovered renal function and survived TA-TMA. Our small cohort demonstrates a trend toward improved renal and overall outcome with the most benefit in patients with multiorgan failure starting TPE as early as 4 days after TA-TMA diagnosis, in conjunction with discontinuation of calcineurin inhibitor and concomitant use of rituximab. While TA-TMA characteristics and degree of organ injury was comparable in survivor and nonsurvivor groups at the time TPE was initiated for TA-TMA diagnosis, one could speculate that a more acute presentation of TA-TMA in certain patients might indicate different disease pathogenesis and response to therapy. It is also plausible that TPE institution early in TA-TMA course is able to abort the pathologic process contributing to the endothelial injury, while patients with more advanced TA-TMA are not able to recover from vascular injury even if the microangiopathic process ceases with prolonged TPE. Recently, TPE was also reported to be beneficial in previously healthy pediatric patients with fulminant sepsis-induced multiorgan failure, if instituted early (within hours) in the course of disease. These patients were thought to have component of sepsis-induced TMA and cytokine storm contributing to acute organ injury that was corrected by TPE. ${ }^{23}$ However, the mechanism of action of TPE, whether removal of inflammatory mediators involving endothelial activation and microvascular thrombosis, remains to be investigated.

The strengths of our report include the use of uniform diagnostic TA-TMA criteria and a standardized therapeutic approach including reduction or elimination of calcineurin inhibitor exposure and administration of rituximab in all study patients reducing confounding variables and allowing better assessment of TPE effect. The TPE procedure itself was well tolerated without any significant procedure-related complications. It is important to emphasize that insertion of a large central catheter for vascular access in 
immunocompromised and thrombocytopenic pediatric patients is not without risk but likely justified in patients suffering from multiorgan impairment due to TA-TMA. As outlined below, future research should focus on the pathophysiology and early diagnosis of TA-TMA, to best determine which patients would benefit from the early initiation of TPE.

Several limitations of our study deserve attention. Our cohort is small, preventing us from detecting small changes in outcome between the groups. Given the retrospective acquisition of data, we cannot exclude the possibility that selection bias was responsible for our favorable results. Therefore, we cannot conclude that TPE alone was responsible for the improved survival in our patients who started therapy earlier.

There are no universally accepted guidelines for the use of TPE in TA-TMA. Our current practice is to closely monitor for TA-TMA after HSCT with twice weekly LDH measurements and close attention to blood pressure and renal function, including urinalysis findings of unexplained proteinuria. Those having concern for TA-TMA are further evaluated for microangiopathic hemolysis by checking serum haptoglobin weekly and reviewing the daily peripheral smear blood smear for schistocytes. Strong consideration is given to confirming the diagnosis histologically with a kidney biopsy in those with a favorable risk-benefit profile for this procedure. Patients diagnosed with TA-TMA have their calcineurin inhibitor exposure reduced or discontinued, if clinically feasible, and are changed to alternative GVHD prophylaxis, such as MMF and/or steroids. Potential viral triggers for TA-TMA are identified and treated aggressively. Patients with TA-TMA without multiorgan failure are given two weekly doses of rituximab $\left(375 \mathrm{mg} / \mathrm{m}^{2} / \mathrm{dose}\right)$ and are reevaluated for clinical response. Those with multiorgan failure, severe hypertension (including hypertensive encephalopathy), or acute kidney injury attributed to TA-TMA are offered first-line therapy with TPE. As mentioned, TPE is performed daily using a singlevolume plasma exchange and continued until resolution of microangiopathy and then tapered to every other day for approximately 2 weeks, twice a week for a week, and then discontinued. Daily TPE is resumed if laboratory findings indicate recurrence of microangiopathy. Priming is done with RBCs for patients weighing less than $21 \mathrm{~kg}$. Replacement fluid is calculated based on the patient's total blood volume. FFP is replaced at $100 \%$ leaving the patient euvolemic at the end of the procedure. Anticoagulation is achieved using citrate. Prophylactic infusion of calcium gluconate is administered for the patient to avoid citrate toxicity. For patients receiving rituximab during TPE therapy, it is given immediately after TPE procedure to provide adequate exposure. TA-TMA is considered TPE refractory if microangiopathy is not controlled in 4 weeks with daily TPE sessions.

More research is needed to determine the exact pathogenesis of TA-TMA and its mechanisms of injury so that these therapies can be assessed more rationally. Along these lines, several studies have reported on potential TA-TMA-associated variables that may predict an individual patient's response to TPE. These predictors have included the use of a TA-TMA index, the LDH level, the development of acute GVHD, and the ability to clear circulating endothelial particles. ${ }^{18,24,25}$ It is interesting to note that prompt initiation of TPE has become an accepted therapeutic option in patients developing DEAP-HUS (deficiency of CFHR [complement factor H-related] plasma proteins and autoantibody-positive form of hemolytic uremic syndrome). ${ }^{11,12}$ The pathologic similarities between TA-TMA and aHUS, 
along with emerging evidence on the potential role of complement system involvement in TA-TMA ${ }^{4,15}$ require further study for customization of therapy options.

\title{
ABBREVIATIONS
}

\author{
aHUS atypical hemolytic uremic syndrome \\ ESRD end-stage renal disease \\ HSCT(s) hematopoietic stem cell transplant(s) \\ MMF mycophenolate mofetil \\ nucGFR nuclear glomerular filtration rate \\ TA-TMA hematopoietic stem cell transplant-associated thrombotic microangiopathy \\ TPE therapeutic plasma exchange \\ TTP thrombotic thrombocytopenic purpura
}

\section{References}

1. George JN. Hematopoietic stem cell transplantation-associated thrombotic microangiopathy: defining a disorder. Bone Marrow Transplant. 2008; 41:917-8. [PubMed: 18545227]

2. Batts ED, Lazarus HM. Diagnosis and treatment of transplantation-associated thrombotic microangiopathy: real progress or are we still waiting? Bone Marrow Transplant. 2007; 40:709-19. [PubMed: 17603513]

3. Changsirikulchai S, Myerson D, Guthrie KA, McDonald GB, Alpers CE, Hingorani SR. Renal thrombotic microangiopathy after hematopoietic cell transplant: role of GVHD in pathogenesis. Clin J Am Soc Nephrol. 2009; 4:345-53. [PubMed: 19144762]

4. Laskin BL, Goebel J, Davies SM, Jodele S. Small vessels, big trouble in the kidneys and beyond: hematopoietic stem cell transplant associated-thrombotic microangiopathy. Blood. 2011; 118:145262. [PubMed: 21596850]

5. Kim JJ, Goodship TH, Tizard J, Inward C. Plasma therapy for atypical haemolytic uraemic syndrome associated with heterozygous factor H mutations. Pediatr Nephrol. 2011; 26:2073-6. [PubMed: 21717289]

6. Polito MG, Kirsztajn GM. Thrombotic microangiopathies: thrombotic thrombocytopenic purpura/ hemolytic uremic syndrome. J Bras Nefrol. 2010; 32:303-15. [PubMed: 21103695]

7. Colic E, Dieperink H, Titlestad K, Tepel M. Management of an acute outbreak of diarrhoeaassociated haemolytic uraemic syndrome with early plasma exchange in adults from southern Denmark: an observational study. Lancet. 2011; 378:1089-93. [PubMed: 21871657]

8. Ho VT, Cutler C, Carter S, Martin P, Adams R, Horowitz M, Ferrara J, Soiffer R, Giralt S. Blood and marrow transplant clinical trials network toxicity committee consensus summary: thrombotic microangiopathy after hematopoietic stem cell transplantation. Biol Blood Marrow Transplant. 2005; 11:571-5. [PubMed: 16041306]

9. Rock GA, Shumak KH, Buskard NA, Blanchette VS, Kelton JG, Nair RC, Spasoff RA. Comparison of plasma exchange with plasma infusion in the treatment of thrombotic thrombocytopenic purpura. Canadian Apheresis Study Group. N Engl J Med. 1991; 325:393-7. [PubMed: 2062330]

10. Uderzo C, Bonanomi S, Busca A, Renoldi M, Ferrari P, Iacobelli M, Morreale G, Lanino E, Annaloro C, Volpe AD, Alessandrino P, Longoni D, Locatelli F, Sangalli H, Rovelli A. Risk factors and severe outcome in thrombotic microangiopathy after allogeneic hematopoietic stem cell trans-plantation. Transplantation. 2006; 82:638-44. [PubMed: 16969286] 
11. Abarrategui-Garrido C, Martinez-Barricarte R, Lopez-Trascasa M, de Cordoba SR, Sanchez-Corral P. Characterization of complement factor H-related (CFHR) proteins in plasma reveals novel genetic variations of CFHR1 associated with atypical hemolytic uremic syndrome. Blood. 2009; 114:4261-71. [PubMed: 19745068]

12. Zipfel PF, Mache C, Muller D, Licht C, Wigger M, Skerka C. DEAP-HUS: deficiency of CFHR plasma proteins and autoantibody-positive form of hemolytic uremic syndrome. Pediatr Nephrol. 2010; 25:2009-19. [PubMed: 20157737]

13. Au WY, Ma ES, Lee TL, Ha SY, Fung AT, Lie AK, Kwong YL. Successful treatment of thrombotic microangiopathy after haematopoietic stem cell transplantation with rituximab. Br J Haematol. 2007; 137:475-8. [PubMed: 17433026]

14. Carella AM, D’Arena G, Greco MM, Nobile M, Cascavilla N. Rituximab for allo-SCT-associated thrombotic thrombocytopenic purpura. Bone Marrow Transplant. 2008; 41:1063-5. [PubMed: 18317457]

15. Jodele S, Bleesing JJ, Mehta PA, Filipovich AH, Laskin BL, Goebel J, Pinkard SL, Davies SM. Successful early intervention for hyperacute transplant-associated thrombotic microangiopathy following pediatric hematopoietic stem cell transplantation. Pediatr Transplant. 2012; 16:E39-42. [PubMed: 21054715]

16. Nguyen L, Terrell DR, Duvall D, Vesely SK, George JN. Complications of plasma exchange in patients treated for thrombotic thrombocytopenic purpura. IV. An additional study of 43 consecutive patients, 2005 to 2008. Transfusion. 2009; 49:392-4. [PubMed: 19389220]

17. Worel N, Greinix HT, Leitner G, Mitterbauer M, Rabitsch W, Rosenmayr A, Hocker P, Kalhs P. ABO-incompatible allogeneic hematopoietic stem cell transplantation following reduced-intensity conditioning: close association with transplant-associated microangiopathy. Transfus Apher Sci. 2007; 36:297-304. [PubMed: 17569587]

18. Cho BS, Min CK, Eom KS, Kim YJ, Kim HJ, Lee S, Cho SG, Kim Y, Kim DW, Lee JW, Min WS, Kim CC. Clinical impact of thrombotic microangiopathy on the outcome of patients with acute graft-versus-host disease after allogeneic hematopoietic stem cell transplantation. Bone Marrow Transplant. 2008; 41:813-20. [PubMed: 18209725]

19. Cho BS, Yahng SA, Lee SE, Eom KS, Kim YJ, Kim HJ, Lee S, Min CK, Cho SG, Kim DW, Lee JW, Min WS, Park CW. Validation of recently proposed consensus criteria for thrombotic microangiopathy after allogeneic hematopoietic stem-cell transplantation. Transplantation. 2010; 90:918-26. [PubMed: 20717073]

20. Darabi K, Berg AH. Rituximab can be combined with daily plasma exchange to achieve effective B-cell depletion and clinical improvement in acute autoimmune TTP. Am J Clin Pathol. 2006; 125:592-7. [PubMed: 16627268]

21. National High Blood Pressure Education Program Working Group on High Blood Pressure in Children and Adolescents. The fourth report on the diagnosis, evaluation, and treatment of high blood pressure in children and adolescents. Pediatrics. 2004; 114:555-76. Suppl 4th Report. [PubMed: 15286277]

22. Laskin BL, Goebel J, Davies SM, Khoury JC, Bleesing JJ, Mehta PA, Filipovich AH, Paff ZN, Lawrence JM, Yin HJ, Pinkard SL, Jodele S. Early clinical indicators of transplant-associated thrombotic microangiopathy in pediatric neuroblastoma patients undergoing auto-SCT. Bone Marrow Transplant. 2011; 46:682-9. [PubMed: 20697372]

23. Qu L, Kiss JE, Dargo G, Carcillo JA. Outcomes of previously healthy pediatric patients with fulminant sepsis-induced multisystem organ failure receiving therapeutic plasma exchange. J Clin Apher. 2011; 26:208-13. [PubMed: 21786317]

24. Erdbruegger U, Woywodt A, Kirsch T, Haller H, Haubitz M. Circulating endothelial cells as a prognostic marker in thrombotic microangiopathy. Am J Kidney Dis. 2006; 48:564-70. [PubMed: 16997052]

25. Kennedy GA, Kearey N, Bleakley S, Butler J, Mudie K, Durrant S. Transplantation-associated thrombotic microangiopathy: effect of concomitant GVHD on efficacy of therapeutic plasma exchange. Bone Marrow Transplant. 2010; 45:699-704. [PubMed: 19767787] 


\section{TABLE 1}

Patient demographics and treatment characteristics *

\begin{tabular}{|c|c|c|c|}
\hline Patients & Survived $(\mathrm{n}=5)$ & $\operatorname{Died}(n=5)$ & p value \\
\hline Age at time of HSCT & $3(3,7)(2-16)$ & $6(4,18)(1-22)$ & 0.46 \\
\hline \multicolumn{4}{|l|}{ Underlying diagnosis } \\
\hline Malignancy & $4(80)$ & $1(20)$ & 0.21 \\
\hline Immunodeficiency & $1(20)$ & $3(60)$ & 0.52 \\
\hline Bone marrow failure & $0(0)$ & $1(20)$ & 1.0 \\
\hline HSCT type & & & 1.0 \\
\hline Allogeneic & $3(60)$ & $4(80)$ & \\
\hline URD & $2 / 3$ & $4 / 4$ & 0.43 \\
\hline MSD & $1 / 3$ & $0 / 4$ & 0.43 \\
\hline Autologous & $2(40)$ & $1(20)$ & 1.0 \\
\hline Conditioning regimen & & & 0.42 \\
\hline Myeloablative & $4(80)$ & $3(60)$ & \\
\hline Reduced intensity & $1(20)$ & $2(40)$ & \\
\hline Pre-HSCT nucGFR (ml/min/1.73 m²) & $167(137,168)(113-204)$ & $121(78,142)(71-165)$ & 0.12 \\
\hline Proteinuria before HSCT & $0(0)$ & $1(20)$ & 1.0 \\
\hline Creatinine before HSCT (mg/dL) & $0.4(0.3,0.4)(0.3-0.5)$ & $0.5(0.3,0.6)(0.3-1.1)$ & 0.39 \\
\hline \multicolumn{4}{|l|}{ Infections preceding TA-TMA } \\
\hline Viremia & $4(80)$ & $5(100)$ & 1.0 \\
\hline Bacteremia & $0(0)$ & $0(0)$ & 1.0 \\
\hline Fungal infection & $0(0)$ & $0(0)$ & 1.0 \\
\hline
\end{tabular}

Data are presented as median (interquartile range) (range) or number $(\%)$.

$\mathrm{MSD}=$ matched sibling donor; $\mathrm{URD}=$ unrelated donor. 


\section{TABLE 2}

TA-TMA features and response to therapy*

\begin{tabular}{|c|c|c|c|}
\hline Patients & Survived $(\mathrm{n}=5)$ & Died $(\mathbf{n}=5)$ & p value \\
\hline Day after HSCT of TA-TMA diagnosis & $35(28,36)(9-41)$ & $47(26,92)(25-240)$ & 0.35 \\
\hline Maximum fold elevation of pre-BMT creatinine & $10.2(1.6,10.3)(1.5-13.0)$ & $4.8(3.6,5.4)(2.0-16.6)$ & 0.92 \\
\hline Urine protein/creatinine ratio & $13.0(2.4,13.0)(2.4-13.0)^{22}$ & $20.0(5.0,95.5)(2.0-159.0)^{22}$ & 0.72 \\
\hline Rituximab doses & $5(4,7)(4-7)$ & $10(9,16)(4-17)$ & 0.07 \\
\hline Days to initiation of TPE after TA-TMA diagnosis & $17(5,25)(4-25)$ & $32(17,57)(17-73)$ & 0.11 \\
\hline Number of TPE sessions to resolution of microangiopathy & $25(23,26)(17-32)$ & $69(42,75)(38-79)$ & 0.01 \\
\hline Resolution of microangiopathy & $5 / 5$ & $4 / 5$ & 1.00 \\
\hline Number of days to resolve microangiopathy after starting TPE & $14(14,15)(10-16)$ & $54(38,67)(32-70) ; n=4$ & $0.004^{\dagger}$ \\
\hline Complete resolution of TA-TMA & $5 / 5$ & $0 / 5$ & 0.01 \\
\hline Number of RRT sessions to resolution of renal failure & $19(7,30)(7-30) ; n=3$ & $98(64,154)(43-199) ; n=4$ & $0.03^{\dagger}$ \\
\hline $\begin{array}{l}\text { Number of days for creatinine to return to pre-HSCT baseline } \\
\text { after starting TPE }\end{array}$ & $40(32,54)(15-127)$ & Never normal until death & $0.004^{t}$ \\
\hline Recovery of renal function by nucGFR $\left(\mathrm{mL} / \mathrm{min} / 1.73 \mathrm{~m}^{2}\right)$ & $95(68,148)(65-171) ; n=4$ & $\begin{array}{l}\text { None of patients recovered from } \\
\text { ESRD }\end{array}$ & NA \\
\hline
\end{tabular}

* Data are presented as median (interquartile range) (range) or number $(\%)$. None of the p values are corrected for multiple testing.

tTwo in the "survived" group and one in the "died" group did not get RRT.

${ }^{t}$ Analyzed using life table methods and Wilcoxon test as for one death microangiopathy was not resolved and those that died never returned to preBMT levels of creatinine. In each case these patients were censored at death.

$\mathrm{RRT}=$ renal replacement therapy. 